\title{
Crystal Structure and Activity Of Kunjin Virus Ns3 Helicase; Protease and Helicase Domain Assembly in the Full Length Ns3 Protein
}

\author{
M. Milani, E. Mastrangelo, P.V. Konarev ${ }^{\dagger}$, D. Svergun ${ }^{\dagger}$ and M. Bolognesi \\ Dept of Biomolecular Sciences and Biotechnology, and CNR-INFM, University of Milano, I-20131 Milano, Italy \\ ${ }^{1}$ European Molecular Biology Laboratory, Hamburg Outstation, 22603 Hamburg, Germany, and Institute of \\ Crystallography, Russian Academy of Sciences, 117333 Moscow, Russia
}

Flaviviral NS3 is a multifunctional protein displaying $\mathrm{N}$-terminal protease activity in addition to C-terminal helicase, nucleoside 5'-transferase (NTPase), and 5'-terminal RNA triphosphatase (RTPase) activities. NS3 is held to support the separation of RNA daughter and template strands during viral replication.

We solved the three-dimensional structure (at $3.1 \AA$ resolution) of the NS3 helicase domain (residues 186-619; NS3:186-619) from Kunjin virus, an Australian variant of the West Nile virus (1). We showed that NS3:186-619 displays both ATPase and RTPase activity, that it can unwind a double-stranded RNA substrate, being however inactive on a double-stranded DNA substrate. Analysis of different constructs shows that full length NS3 displays increased helicase activity, suggesting that the protease domain plays an assisting role in the RNA unwinding process. The structural interaction between the helicase and protease domain has been assessed using small angle X-ray scattering on full length NS3, disclosing that the protease and helicase domains build a rather elongated molecular assembly differing from that observed in the NS3 protein from hepatitis C virus.

S.A.X.S. data were collected on the X33 camera of the EMBL on the storage ring DORIS III (DESY, Hamburg, Germany). The data were recorded using a MAR345 two-dimensional image plate detector at a sample-detector distance of $2.7 \mathrm{~m}$ and a wavelength of $\lambda=1.5 \AA$, covering the range of momentum transfer $0.012<s<0.45 \AA^{-1}(s=4 \pi \sin \theta / \lambda$, where $2 \theta$ is the scattering angle). The solutions containing NS3FL at 3.0, 1.5 and $0.75 \mathrm{mg} / \mathrm{ml}$ in Hepes $10 \mathrm{mM}, \mathrm{NaCl} 300 \mathrm{mM}$ and protease inhibitors cocktail (Roche), with the addition of $2 \mathrm{mM}$ DTT to reduce radiation damage, were placed in a $70 \mu \mathrm{l}$ cell with polystyrene windows and exposed to X-ray beam. To check for radiation damage, the data were collected in two successive 2-minute exposures, and no changes were observed in the scattering patterns with time, i.e., there was no measurable radiation damage. All measurements were performed at $15^{\circ} \mathrm{C}$. The data were averaged after normalization to the intensity of the incident beam and the scattering of the buffer was subtracted. The difference curves were scaled for the solute concentration and extrapolated to infinite dilution. All data manipulations were performed using the program package PRIMUS (2).

The forward scattering $I(0)$ and the radius of gyration $\left(\mathrm{R}_{\mathrm{g}}\right)$ were evaluated using the Guinier approximation (3); the maximum diameter $D_{\max }$ of the particle were computed from the entire scattering patterns using program GNOM (4), and the excluded volume $V_{p}$ of the particle was computed from the Porod invariant (5). The molecular masses of the solutes were evaluated by comparison of the forward scattering with that from reference solutions of bovine serum albumin $(66 \mathrm{kDa})$. The ab initio shape reconstruction was performed using the program GASBOR (6), using the total number of the residues in NS3FL (619).

The estimated molecular mass of the NS3FL sample, $65 \pm 5 \mathrm{kDa}$, is compatible with monomeric protein (expected mass of $68.9 \mathrm{kDa}$ ). This finding is further corroborated by the excluded volume of the particle, $(155 \pm 10) \times 10^{3} \AA^{3}$, noting that for sufficiently large globular 
proteins the hydrated volume in $\AA^{3}$ should numerically be about twice the moeculra mass expressed in $\mathrm{Da}$. The experimental radius of gyration $\mathrm{R}_{\mathrm{g}}$ and maximum size $\mathrm{D}_{\max }(3.31 \pm 0.05 \mathrm{~nm}$ and $12 \pm 1$ $\mathrm{nm}$, respectively) point to an extended particle structure. Moreover, the distance distribution function of NS3FL has a skewed appearance typical of elongated particles; it also displays two maxima, which is characteristic of particles containing well separated domains.

The SAXS data were used to reconstruct the shape of NS3FL ab initio at low resolution using the program GASBOR (6). Ten independent simulated annealing runs yielded superimposable results neatly fitting the experimental data with discrepancy in the range $\chi=$ $1.41 \div 1.52$ (Fig.5A). The most typical ab initio model of NS3FL displays an elongated particle with two separated domains of unequal size. Such structure can be divided in two main regions: a three lobed structure with a central hollow cavity and an additional elongated 'head' domain. Such a modular envelop can be easily fitted by the three helicase domains and by the DENV protease domain as model for the $\mathrm{N}$-terminal domain, that falls in a region neighboring the $\mathrm{N}$-terminus of the fitted NS3:186-619 structure.

Nevertheless, since the SAXS model does not allow to infer fine structural details, the present data do not provide hints for a mechanism explaining the higher RNA unwinding efficiency observed in NS3FL, relative to the isolated helicase component. Such increased efficiency imply the participation of the protease domain in promoting the helicase activity, and might suggest that further domain rearrangements, affecting the protease/helicase contact interface, may occur in the RNA-bound protein.

\section{References}

[1] Mastrangelo E, Milani M, Bollati M, Selisko B, Peyrane F, Pandini V, Sorrentino G, Canard B, Konarev PV, Svergun DI, de Lamballerie X, Coutard B, Khromykh AA, Bolognesi M. (2007) 372, 444.

[2] Konarev, V., Volkov, V.V., Sokolova, A.V. Koch, M.H.J. and Svergun, D.I. (2003). 33, 1277.

[3] Guinier, A. (1939) 12, 161.

[4] Svergun, D. I. (1992) 25, 495.

[5] Porod, G. (1982). Small-angle X-ray scattering, edited by O. Glatter \& O. Kratky, pp. 17-51. London: Academic Press

[6] Svergun, D.I., Petoukhov, M.V. and Koch, M.H.J. (2001) 80, 2946. 